Jul 1st, 12:00 AM

\title{
Modelling the effect of information feedback on the spread of the Ebola virus
}

Bernadette O’Regan

Richard Moles

Follow this and additional works at: https://scholarsarchive.byu.edu/iemssconference

O’Regan, Bernadette and Moles, Richard, "Modelling the effect of information feedback on the spread of the Ebola virus" (2002). International Congress on Environmental Modelling and Software. 45.

https://scholarsarchive.byu.edu/iemssconference/2002/all/45

This Event is brought to you for free and open access by the Civil and Environmental Engineering at BYU ScholarsArchive. It has been accepted for inclusion in International Congress on Environmental Modelling and Software by an authorized administrator of BYU ScholarsArchive. For more information, please contact scholarsarchive@byu.edu, ellen_amatangelo@byu.edu. 


\title{
Modelling the effect of information feedback on the spread of the Ebola virus
}

\author{
Bernadette O'Regan, Richard Moles \\ Centre for Environmental Research, University of Limerick, Limerick, Ireland (bernadette.oregan@ul.ie)
}

\begin{abstract}
This paper describes the application of the tools and techniques of the system dynamics method to the complex problem of understanding the spread of the Ebola virus. The main deliverable of this research is a computer simulation model in the system dynamics tradition. The essence of system dynamics is to act as a framework for formalising mental models of a problem. In this respect, the system dynamics simulation model presented here is a theory describing the structure of, and interrelationships between, the factors that impact an outbreak of the Ebola virus and the attempts to contain it.

The model, comprising 57 interrelated variables, is structured to represent a group of rural villages served by one local hospital, remote from regional and national medical laboratories. Such a structure typifies the circumstances of recent Ebola outbreaks in central Africa. Model output examines the probable impacts of changes in the system delays. These delays consist mainly of incubation delays, delays to disease recognition, delays in travelling to hospital, delays to inform higher health authorities and delays to involve the Centre for Disease Control in the US.
\end{abstract}

Keywords: Ebola, Computer Simulation, Modelling, System Dynamics, Feedback, Information Delays

\section{INTRODUCTION}

The Ebola virus takes its name from the Ebola River in northern Congo, where it first emerged in 1976. In this outbreak, 318 people were infected and 280 died (Le Guenno and Galabru, 1997). On average the disease has an $80 \%$ fatality rate (Michie, 1999, Peters, 1997). Recent studies (Le Guenno and Galabru, 1997) have identified four strains of the virus, suggested by differences in mortality and clinical expression.

The contamination risk is related to contact with the body fluids of an infected person. This is most likely during the nursing of patients and the preparation of corpses for burial. Also, as the disease kills so rapidly the risk of an epidemic in developed countries is believed to be low (Sinha and Powell, 1996).

Outbreaks tend to spread from one isolated case and so individual behaviour and local customs play an important role in the progress of each outbreak of Ebola or a similar epidemic (Le Guenno and Galabru, 1997). Initial cases leading to secondary transmission to the person taking care of the infected person are called 'familial outbreaks'. The tendency so far has been for the spread to 'amplify' when infected people enter local hospital care without proper protective equipment or hygiene rules and then 'explode' as the virus spreads to general hospitals, as was the case in the Kitwit (Zaire) outbreak in 1995 (Shears, 2000, Peters, 1997). These hospitals tend to be ideal breeding grounds for disease due to the poor training of staff, inadequate staff levels, poor standards of hygiene, lack of analytical laboratory equipment and only basic medicine (Shears, 2000).

\section{AVAILABILITY OF DATA}

Recent outbreaks have confirmed data already acquired by the World Health Organisation (WHO) in 1996. Transmission is not airborne. It requires close contact to a seriously ill patient that, in most situations, results in a low infectiousness rate. Therefore, isolation precautions and a change in cultural customs can result in the containment of the virus. The incubation period varies from 3 days and 3 weeks and when the patient has a lethal form of the disease, death usually takes place 
between 6 and 10 days from the initial onset of the illness (Le Guenno and Galabru, 1997).

The best currently available diagnosis techniques, antigen capture on serum samples, require at least 3 days under the best conditions. This test can only be carried out at biosafety level 4 laboratories.

Little if anything is yet known about the host of the virus, except for the curious fact that a number of outbreaks have occurred around October or November, suggesting a link to seasonal or annual cycles in the forests of central Africa. The recent Ugandan outbreak is consistent with this pattern. The WHO are presently coordinating a project which facilitates the observation and capture of species in chimpanzee territories of the Tai forest, where the chimpanzee population has already been hit by two separate outbreaks of the virus. The remainder of this paper describes a simulation model designed to capture the important dynamics of an Ebola outbreak. The model is developed in the system dynamics tradition, with emphasis on the importance of feedback and information delays.

\section{MODELLING AN OUTBREAK}

For the purposes of the model, it is assumed that the outbreak originates from a single isolated incident on day 15 of the 150 day simulation period. From Figure 1 it can be seen that this random infection resulted in a total death toll of 192 people over the 150 days.
Figure 2 shows the behaviour over time graphs for the spread of the virus in the local village. Note that the number of sick people declines briefly around day 40. This corresponds to the time when the sick start to move into hospital.

The model assumes the size of the susceptible population to be 1000 people. This is assumed to correspond to the size of a rural village. The rate

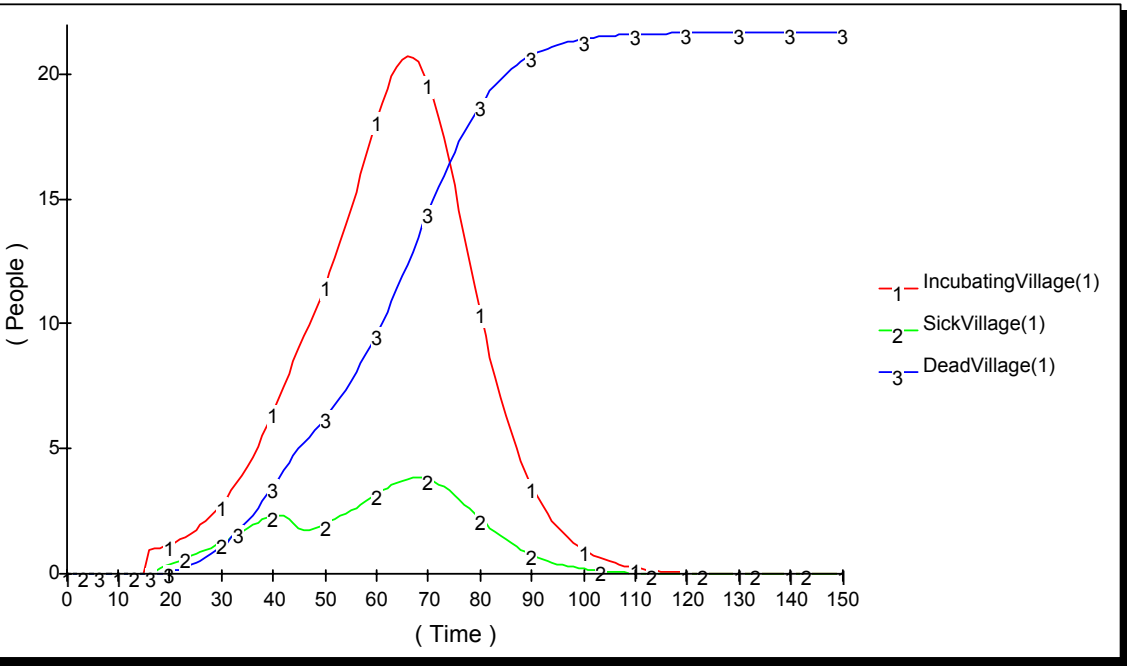

Figure 2. The spread of the virus in the local village

at which other people become infected in the village is a function of how easy it is to become infected (the infectiousness rate), the rate at which an infected person comes in contact with other people (the contact rate) and the percentage of incubating and sick people already in the village, defined as follows;

\section{RandomInfections + (ContactRate InVillage * InfectiousnessInVillage * SusceptibleInVillage *(Incubating InVillage + SickInVillage) / Total Population)}

It is assumed here that both incubating and sick people can pass on the

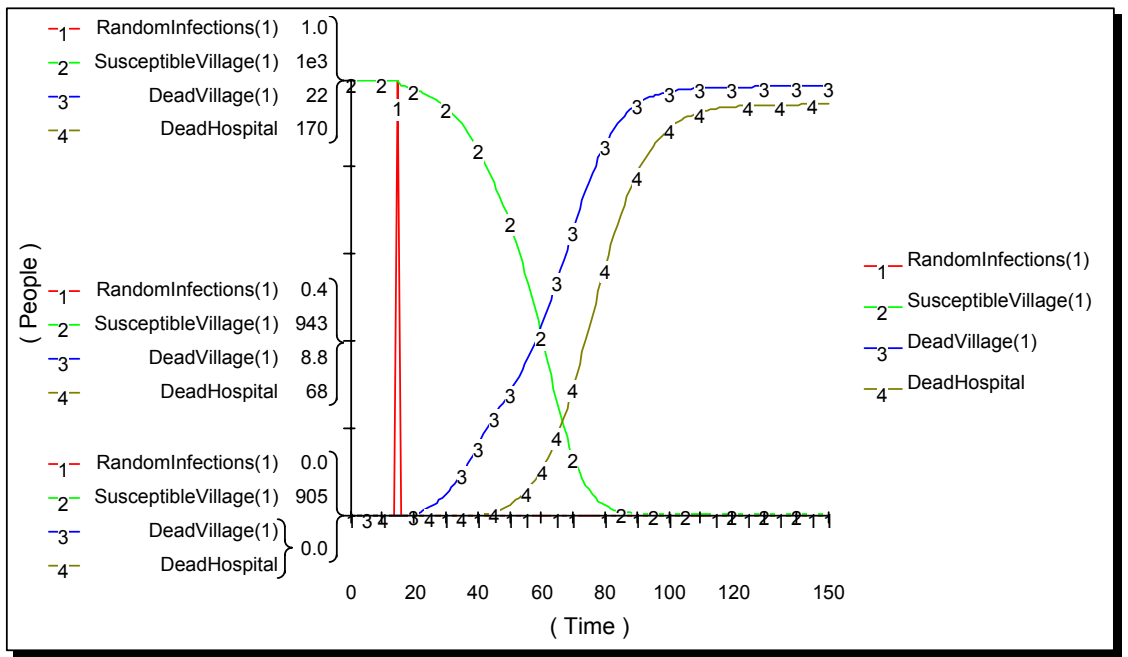
virus. Furthermore, it is assumed that a susceptible person is equally likely to get infected from either an incubating or sick person. In practice, the virus is more likely to be transmitted through the nursing of very sick people but it could be argued that the contact rate would probably be higher for incubating people. In any case, these differences are abstracted away in this model but the model may be

Figure 1. Output from the standard run of the model 
further enhanced if it was ever necessary to disaggregate these relationships.

ContactRateInVillage and InfectiousnessInVillage are more difficult to quantify, however, these variables are important so they must be included in the model. It is also necessary to estimate how many new people might an infected person living in a remote African village meet each day. The standard run of the model as shown in Figure 1 assumes a contact rate of 1 , i.e. an infected person meets one new person each day. However, evidence suggests that once the cause of the epidemic is identified exposed populations are warned of the dangers of coming in contact with infected persons and so the contact is not a constant but instead declines over time and is defined as follows;

\section{ContactRateInVillage $=1 *(1-$ BarrierNursing $)$}

where BarrierNursing, expressed as a percentage, is taken to represent a type of disease containment using isolation techniques implemented by the national disease surveillance unit or the CDC during each Ebola outbreak to date. The InfectiousnessRate, meaning the infectiousness of each contact between a susceptible and an incubating or sick (infectious) person, is defined as 0.15 . This figure was arrived at through extensive sensitivity analysis, based on available data from historical Ebola outbreaks.

A value of 8 days was chosen for the incubation period and the number of people getting sick each day in the village is then defined as;

\section{IncubatingInVillage / \\ IncubationPeriod}

Similarly, DeathsInVillage is defined;

\section{SickInVillage * \\ (DeathFraction / \\ LengthOfSickness)}

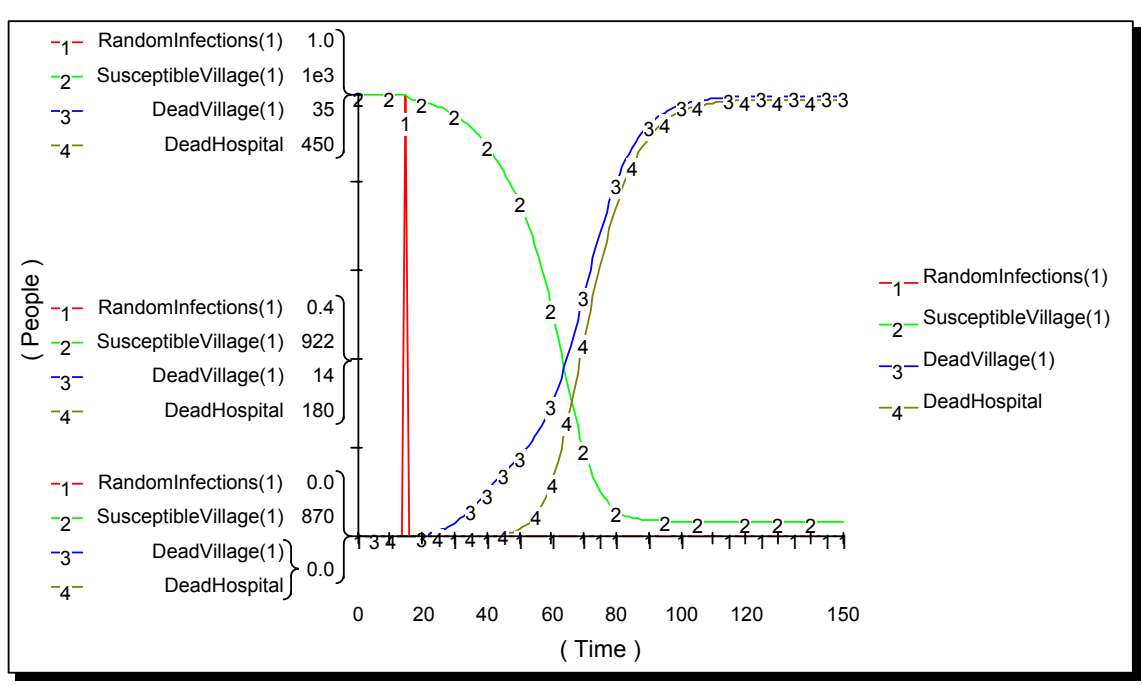

where DeathFraction is assumed to be $80 \%$ and LengthOfSickness to be 6 days.

People who don't die, but remain in the village, become 'survivors', as defined by the SurvivalFraction (1 - DeathFraction). It is assumed for the purposes of this model that survivors remain immune to the virus, at least for the duration of the simulation, i.e. that they do not return to the susceptible population.

3.1 The Local Hospital: Some of the sick people choose to go to the local hospital for treatment. It is assumed that at the start of the epidemic nobody goes to hospital in the belief that the sickness will be treated in the local community. Only when it becomes apparent that the virus cannot be treated locally do people turn to the hospital for help.

TransferRateToHospital is defined as;

\section{SickInVillage * LocalAwarenessOfVirus * 0.5}

where it is assumed that at most $50 \%$ of sick people go to hospital and then only when at least 5 local people have already died.

3.2 Exploring Scenarios: As already stated, it is not the contention of the authors that the assumptions put forward in this paper are necessarily correct. Supposing for example, as might be the case in practice, that the infectiousness rate in the hospital is twice that in the local village. Figure 3 shows the outcome of modifying the standard run to reflect this assumption. In this instance, the death rate in the local hospital increases to 450 people.
Figure 3. Higher infectiousness rate in local hospital

Note also that the death rate in the village also increases, to 35 people, even though the infectiousness rate in the village stays the same. This is because people continuously return home to the village after being treated for other illnesses and some of these have become infected during their stay in hospital. 
This last point raises a number of other possibilities. For example, the local hospital serves many villages. Patients can return home to the other villages infected with the virus, thus further spreading the epidemic. The array feature of the Powersim modelling software was used to extend the model to simulate five villages, each of which were served by the same local hospital. For this run, the hospital population was increased to 1000 to reflect the larger catchment area.

Now suppose that the virus mutates and the incubation period increases to 60 days. Under such a scenario, 1500 people would die in hospital over the period of 1 year (see Figure 4) and a further 240 deaths in the villages.

A higher incubation period for such a devastating virus as Ebola could destroy the entire local population were it not for outside intervention. The model could be further expanded to include a regional or national hospital sub-system but again, for simplicity, this was not attempted here.

Instead, HospitalAwarenessOfVirus (modelled on LocalAwarenessOfVirus), is used as a basis for forwarding specimens and clinical data to the regional or national laboratories and ultimately the CDC. There are a number of critical information delays in this process.

An information delay represents the process of a gradual, delayed adjustment of information moving towards the value being supplied by the source. The model considers four (cumulative) sources of delay, namely DelayToAnalysisOfBloodSamples, DelayTo InvolveCDC, DelayInRecognisingEbola and DelayInEnforcementOfBarrierNursing.

It is known from experience that it takes 5 days for the CDC to diagnose Ebola. Once diagnosed, it can take up to 1 week for CDC and WHO experts to arrive at the scene of the outbreak, locate all people exposed to the virus and enforce barrier nursing and encourage other protective measures. The delay to analysis of blood samples and the delay to involve the CDC are functions of the number of 'tiers' between the local hospital and the eventual diagnosis of the virus. In some African countries attempts have already been made to improve diagnosis facilities and, in such cases, there may be no need to involve the CDC. However, the standard run of this model assumes a delay of 5 days for each of these variables. The number of decisions to be made (the number of levels of authority to be called upon) impacts the nature of the delays in the system.

The standard run of the model assumes a total information delay of 22 days before barrier nursing was fully in place. Suppose for example that the virus could not be diagnosed or that the CDC did not get involved. In such circumstances, the initial regional population of 6,000 is reduced by $66 \%$. Virtually everyone in hospital is dead. All that prevents total annihilation is the assumption that survivors remain immune.

Now assume that our understanding of the virus improves to the extent that Ebola is diagnosed in the local hospital by the time the 5th patient dies (a very unlikely scenario under current circumstances). Figure 5 shows model output under this scenario.

The 5 th person dies in the local hospital on day 51 . Barrier nursing is enforced with immediate effect.

A further 13 people

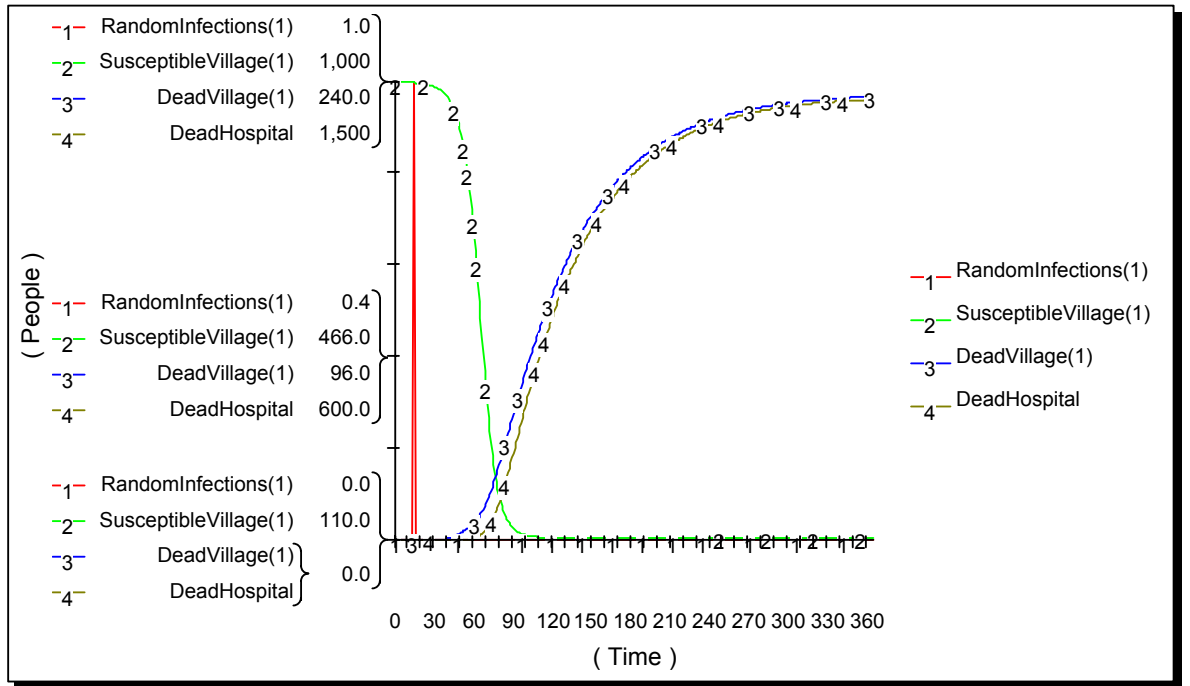

Figure 4. 60 day incubation period simulated over 12 months still die in the hospital, even with the contact rate down to 0 . This is because by the time 5 people have died in hospital, 6 are already sick and a further 4 are incubating. 6 people have died in the villages and a further 7 are incubating the disease by this time. The point here is that although national laboratory or $\mathrm{CDC}$ intervention is critical it is still incapable of preventing further deaths because of the delay between the 1 st and 5 th deaths. 
Following on from this point, consider the scenario whereby the virus can be diagnosed in the local hospital once the first patient becomes sick. Under this scenario, the total death rate is restricted to 10 people (see Figure 6).

\section{CONCLUSIONS}

"System dynamics models do not, of course, permit one to predict the future. Rather, the objective is experimental. It is to assemble the diverse bits of quantitative and qualitative information already available and evaluate which data are the most important in understanding the system as a whole." [Randers,

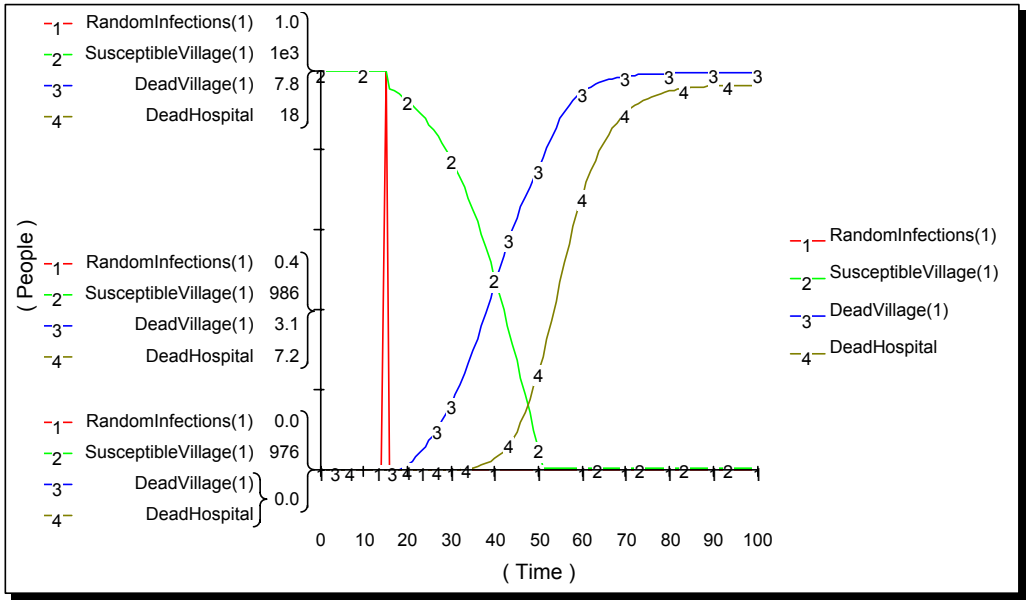

Figure 5. Diagnosis in local hospital 1973, p51]

The system dynamics model described in this paper is structured to represent a group of rural villages served by one local hospital, remote from regional or national laboratories. Such a structure typifies the circumstances of recent Ebola outbreaks in central Africa.

In creating the simulation model described here, the intention is to enrich existing verbal and written models of the factors impacting the spread of the Ebola virus. This model may be thought of as the synthesis of existing documentation to form a more transparent and dynamic tool for hypothesis testing. More specifically;

- Through a quantitative analysis of existing data, the model exposes, within the context of the problem area, the underlying assumptions used as a basis for system understanding. Through the compression of time, the model provides a means of taking these assumptions to their logical conclusions. Exposing assumptions in this way leaves less room for misinterpretation and provides a solid basis for enhancing the understanding of system structure.

- The behaviour-over-time graphs used to

Figure 6. Ebola diagnosed on 1st sickness in hospital

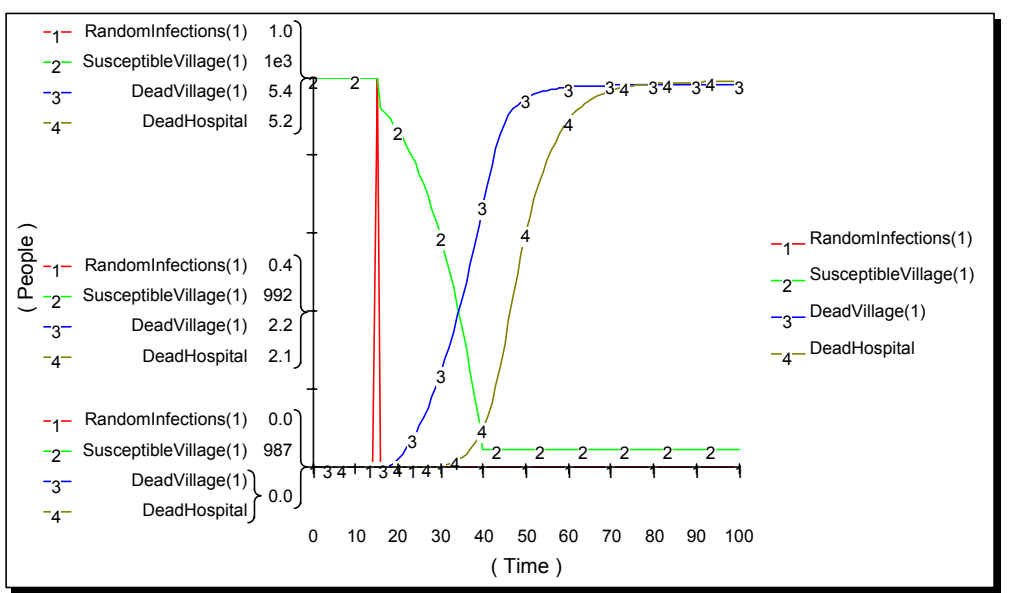
present model output provide a powerful means of exposing system complexity and, thus, increasing understanding.

- The model shows how the feedback mechanisms inherent in system structure influence behaviour patterns over time.

- The assumptions of the authors are made explicit through the structure of the model and the choice of parameter values. The behaviourover-time graphs used to present model output provide a powerful means of exposing system complexity and, thus, increasing understanding. The exact nature of 
this complexity, as assumed by the model, is made explicit through the variable definitions. These definitions can be modified and the resulting changes in behaviour patterns examined.

This model, linked to a GIS, will provide an aid for health workers dealing with the Ebola virus and attempting to estimate the behaviour of a possible epidemic under a variety of situations. This is becoming increasingly important with the growth of travel to these remote regions and with the movement of refugees between countries (Kalipeni and Oppong, 1998, Freedman and Woodall, 1999).

Finally, the model aims to encapsulate best practice in the field of system dynamics. It emphasises the difference between actual and perceived conditions as a basis for action. It makes explicit the underlying assumptions as a basis for further expansion. It highlights system structure as a catalyst for change.

\section{REFERENCES}

Freedman, D.O., Woodall, J., (1999), "Emerging infectious diseases and risk to the traveler", Medical Clinics of North America, Vol. 83, No. 4, pp 865-883.

Kalipeni, E., Oppong, J., (1998), 'The refugee crisis in Africa and implications for health and disease: A political Ecology Approach', Social Science and Medicine, Vol. 46, No. 12, 15 June 1998, pp1637-1653.

Le Guenno, B., Galabru, J., (1997), "Ebola Virus", Bulletin de l'Institut Pasteur, Vol. 95, No. 2, 6 April, pp73-83.

Michie, C., (1999), "Lessons from the survivors of the Ebola infection", Molecular Medicine Today, Vol. 5, No. 7, pp285.

Peters, C.J., (1997), "Ebola and Hanta Viruses", FEMS Immunology and Medical Microbiology, Vol. 18, No. 4, August, pp281-289.

Randers, J., Meadows, D., (1973), "The Dynamics of Solid Waste Generation", Towards Global Equilibrium: Collected Papers, Meadows, D., Meadows, D. (Eds.), Wright-Allen Press, Cambridge, U.S.A., pp165-212.

Shears, P., (2000), "Emerging and reemerging infections in Africa: the need for improved laboratory services and disease surveillance", Microbes and Infection, Vol. 2, No. 5, pp 489-495.
Sinha, G., Powell, C.S., (1996), "Shaking the Ebola tree", Scientific American, August, http://www.sciam.com/explorations/082696explor ations.html.

Vick, K., (2000), Washington Post Foreign Service, October 14, ppA24. 